\title{
The Effect of Selected Chemical Preservatives and Starter Cultures on the Sensory Characteristics and Shelf Life of Rice Injera
}

\author{
Yassin Hassen $^{1^{*}}$, Ivan Muzira Mukisa ${ }^{2}$ and Henok Kurabachew ${ }^{1}$
}

${ }^{1}$ Food Science and Technology, School of Nutrition, College of Agriculture, Hawassa University, Hawassa, Ethiopia

${ }^{2}$ Department of Food Technology and Nutrition, Makerere University, Kampala, Uganda

"Corresponding author: Yassin Hassen, Food Science and Technology, School of Nutrition, College of Agriculture, Hawassa University, Hawassa, Ethiopia, Tel: +251462205311; E-mail: yassin.hassen@gmail.com

Received date: January 18, 2018; Accepted date: February 05, 2018; Published date: February 13, 2018

Copyright: (c) 2018 Hassen Y, et al. This is an open-access article distributed under the terms of the Creative Commons Attribution License, which permits unrestricted use, distribution, and reproduction in any medium, provided the original author and source are credited.

\begin{abstract}
Injera is yeast-risen flat bread with a unique, slightly spongy texture. It is a national staple in Ethiopia that is eaten daily in virtually every household. Rice injera has a very short life (2-3 days) at room temperature and essentially spoils due to mould growth. The use of weak organic acid as preservative is allowed in acidic foods, primarily as mould inhibitors. In this study, the effects of chemical preservatives: benzoic acid, sodium benzoate, and potassium sorbate and starter cultures (Lactobacillus plantarum+Saccharomyces cerevisiae) on the quality and shelf life of rice injera were investigated. Benzoic acid $(0.1 \%)$, sodium benzoate $(0.1 \%)$, potassium sorbate $(0.2 \%)$ and a $1: 1: 1$ blend of the three $(0.2 \%)$ were added immediately before baking. Qualitative descriptive analysis $(n=10)$ was used to describe the characteristics of the injera samples. A consumer acceptability panel $(n=30)$ was used to determine the acceptability and shelf life of injera. The preservatives significantly $(p<0.05)$ prolonged the shelf life of injera for up to 10 days with the combination of the three starters achieving the greatest effect. The findings of this study showed that the rice injera prepared with chemical preservatives $(0.1$ benzoic acid, $0.2 \%$ potassium sorbate, $0.1 \%$ sodium benzoate and $0.2 \%$ of the three preservatives retains maximum overall acceptability. Rice injera produced under controlled fermentation L. plantarum, and S. cerevisiae produce injera with better sensory quality than the traditional by using Irsho. Nine descriptors/attributes describing appearance, aroma/odor, taste, and texture were generated to characterize the sensory properties of rice injera. Softness, rollablity, fluffiness, eye size, whitness of top and bottom surfaces and slight sour taste are main characterstics descriptors of rice injera. Softness, fluffiness, spongness, slight sourness and eye size are attributes associated with quality of rice injera. Therefore, a starter combination of L. plantarum + S. cerevisiae together with $0.2 \%$ of the blend of the preservatives $(0.1 \%$ benzoic acid $+0.1 \%$ sodium sorbate $+0.2 \%$ potassium sorbate) in $1: 1: 1$ ratio preservatives can be used to produce acceptable injera with a shelf life of 10 days at room temperature.
\end{abstract}

Keywords: Rice injera; Sensory attributes; Benzoic acid; Potassium sorbate; Sodium benzoate

\section{Introduction}

Injera is a yeast-risen flatbread with a unique, slightly spongy texture. It is a national staple in Ethiopia that is eaten daily in virtually every household. It can be made from different cereals, including sorghum, teff, corn, wheat, barley, rice or a combination of some of these cereals [1].

The shelf-life of a food is the period for which it remains safe and suitable for consumption. This means that the food has not deteriorated in quality or spoiled in any way that the consumer would find unacceptable. Preservatives are additives that primarily contribute to food safety and the prevention of food spoilage. Food additives are generally added to processed foods to prolong the shelf-life by protecting food against deterioration caused by microorganisms [2]. Some of the widely used chemical preservatives include benzoic and sorbic acids and their salts (sodium benzoate and potassium sorbate) [3]. Although they are effective against a wide range of bacteria, sorbates and benzoates are generally used to inhibit yeast and mould growth. These compounds are most active in low $\mathrm{pH}$ foods and essentially ineffective in foods at neutral $\mathrm{pH}$ values [4-6].
Ashagrie and Abate described the major quality attributes of a good injera as a slightly sour taste, which is due to the acidic (low $\mathrm{pH}$ ) nature of injera. It has been reported that the inclusion of sorbates or benzoates post fermentation and immediately before baking may delay the spoilage of injera due to molding [7].

In a study on effects of preservatives on shelf life of teff injera [8]. It was observed that the effectiveness of preservation was ranked as sodium benzoate $>$ benzoic acid $>$ potassium sorbate $>$ blend of (sodium benzoate, benzoic acid, potassium sorbate and calcium propanoate) $>$ calcium propionate.

Rice injera has a shelf life of only 2-3 days and its spoilage is essentially caused by mould growth. The use of weak organic acid as preservative is allowed in acidic foods, primarily as mould inhibitors. Although the effects of preservatives on the shelf life of injera have been studied [9]. Only teff-based injera was evaluated and furthermore the effect of the preservatives on the sensory attributes was not determined. The sensorial characteristics of rice injera have also hitherto not been described yet these would be vital in developing an industrial process for rice injera production. Therefore the aim of this study was to describe the sensorial characteristics of rice injera, as well as to determine the effect of preservatives (benzoic acid, potassium sorbate, sodium benzoate and their combinations) and starters 
(Lactobacillus plantarum + Saccharomyces cerevisiae) on the sensory characteristics and shelf life of rice injera.

\section{Materials and Methods}

\section{Preparation and fermentation of rice batter}

Rice, wheat flour and maize samples were bought from Hawassa open market in Ethiopia. The rice and maize were cleaned of dust, other seeds and foreign matter. The samples were ground into flour at a local flour mill.

Injera batter was prepared by mixing the flours (rice:wheat:maize in a ration of (10:1.5:2) was mixed with water in the proportions of $1: 2$ $(\mathrm{w} / \mathrm{v})$ flours to water in $5 \mathrm{~L}$ plastic bucket. A mixed culture of Lactobacillus plantarum and Saccharomyces cerevisiae was inoculated into the batter in two different plastic buckets. The inoculation yielded an initial concentration of $6.6 \pm 0.2 \mathrm{log} \mathrm{cfu} \mathrm{g}^{-1}$ of Lactobacillus plantarum and $4.5 \pm 0.3 \mathrm{log} \mathrm{cfu} \mathrm{g}^{-1}$ of Saccharomyces cerevisiae at the start of the fermentation. Previously fermented injera batter (10\%) was used as a starter for the traditional fermentation (Control). The injera batter was fermented at room temperature (about $25^{\circ} \mathrm{C}$ ) for 24 and 48 h. After fermentation the batters were divided into 5 portions and preservative was added prior to baking. The preservative treatments used include: $0.1 \%$ benzoic acid, $0.1 \%$ sodium benzoate, $0.2 \%$ potassium sorbate and $0.2 \%$ of the blend of the three preservatives ( $0.1 \%$ benzoic acid, $0.1 \%$ sodium benzoate, $0.2 \%$ potassium sorbate in a ratio of 1:1:1).

\section{Descriptive sensory analysis}

A sensory panel $(n=10)$ comprising of students of Hawassa University as well as people from surrounding areas was trained in qualitative descriptive analysis (QDA). Panel training encompasses term generation, concept alignment and panel testing phases. Panel performance was evaluated following guidelines given by Cross [11]. A vocabulary of sensory attributes (descriptors) was developed using a variety of traditionally processed and chemically preserved fermented rice injera (Table 1). A score sheet was prepared using the selected descriptors. Each attribute was evaluated using a 10-point numerical scale (0-9) anchored on both sides with verbal descriptions (e.g., $0=$ not white, $9=$ very white) to allow the panel to score the intensity of the attributes on a framed common scale. The descriptive analysis of each rice injera sample was done in triplicate. Assessment was done in individual booth. During this stage, rice injera samples were presented to the panelists. Commercial bottled water was provided for rinsing the mouth between samples.

\section{Consumer acceptability}

An untrained consumer panel $(n=30)$ comprising of students and members of Hawassa University, as well as other people from surrounding areas were used. Panellists ranked the acceptability of various attributes of the different rice injera samples using a 9-point hedonic scale (9="like extremely"; 8="like very much"; 7="like moderately"; 6="like slightly"; $5=$ "neither like nor dislike"; $4=$ "dislike slightly"; 3="dislike moderately"; 2="dislike very much"; 1="dislike extremely"). The samples were presented on identical serving trays and coded with three digits random numbers. The order of sample presentation was randomized. Clean mineral water held at room temperature was served for cleansing the palate.

\section{Effect of chemical preservatives on shelf life and acceptability of rice injera}

Chemical preservatives, $0.1 \%$ benzoic acid, $0.1 \%$ sodium benzoate, $0.2 \%$ potassium sorbate and $0.2 \%$ blend of the three preservatives were added in 1:1:1 ratio to rice batters just immediately before baking [12]. After the baking process, the experimental and control injera samples were stored at room temperature in the laboratory. In this study, shelf life of injera was defined as the time (in days) when mould growth was first observed by the naked eye. To determine this, the injera samples were examined on a daily basis for visible signs of mould growth on the crust. Samples were visually evaluated for mould growth and changes in appearance. Acceptability was determined as described earlier.

\section{Data analysis}

Data of consumer-oriented sensory evaluations is presented as means of three independent fermentations. Means were subjected to one-way analysis of variance (ANOVA) to test for significant differences $(\mathrm{p}<0.05)$ using SAS software (version 9.0, Addinsoft, Paris, France). Comparisons were made using the Fisher's least significant difference (LSD) test to determine which means were significantly different. Shelf life data was analyzed by using descriptive statistics (SPSS version 16.0). Principle component analysis (PCA) was computed for the descriptive data using XLSTAT software (version 2015, Addinsoft, New York, NY).

\section{Results}

\section{Descriptive characteristics of rice injera}

Nine descriptors/attributes describing appearance, aroma/odor, taste, and texture were generated to characterize the sensory properties of rice injera (Table 1). The characteristics include whiteness of the top and bottom surface, eye size, eye distribution, softness, fluffiness, roll ability, aroma and Sourness. Results of sensory descriptive analysis indicated that preservatives influenced the sensory characteristics of rice injera.

\begin{tabular}{|l|l|}
\hline Descriptors & Definition \\
\hline Appearance & Characteristics that encompass all visually perceptible sensory impressions \\
\hline Fluffy & light or airy \\
\hline Spongy & It has a firm, yet well aerated structure, similar to a sea sponge \\
\hline softness & Easy to chew \\
\hline
\end{tabular}


Citation: Hassen Y, Mukisa IM, Kurabachew H (2017) The Effect of Selected Chemical Preservatives and Starter Cultures on the Sensory Characteristics and Shelf Life of Rice Injera. J Bioprocess Biotech 8: 313. doi:10.4172/2155-9821.1000313

Page 3 of 7

\begin{tabular}{|l|l|}
\hline Color & \\
\hline Whitness of the top surface & The intensity of white color \\
\hline Whitness of the bottom surface & The intensity of white color \\
\hline Texture & Structural conformational quality of food that can be assessed, rheological, optical and acoustic methods \\
\hline Rollability & Rolling around without cracking nature of flat bread \\
\hline Eye distribution & How scattered or evenly distributed the pores/eyes are \\
\hline Eye size & How big the pores/eyes are \\
\hline Taste & The range showing the extent of fermentation \\
\hline Sour & on tasting \\
\hline
\end{tabular}

Table 1: Descriptors for rice injera and their definitions.

Principal component analysis (PCA) of the sensory attributes of rice injera (Figure 1) resulted in a two component resolution accounting for $54.07 \%$ of the variation in the data. The first PC explained $36.54 \%$ of the variation while the second explained $17.53 \%$. The black dots shows sensory attributes descriptors of the rice injera while the black triangle shows the different types of rice injera prepared from $L$. plantarum, $S$. cerevisiae and Irsho with the addition $0.1 \%$ benzoic acid, $0.1 \%$ sodium benzoate and $0.2 \%$ potassium sorbate and $0.2 \%$ of the blend of the three preservatives (benzoic acid, sodium benzoate and potassium sorbate) in 1:1:1 ratio. Benzoic acid was associated with flavor and aroma of the rice injera, potassium sorbate was assoiated with sourness and roll ability.

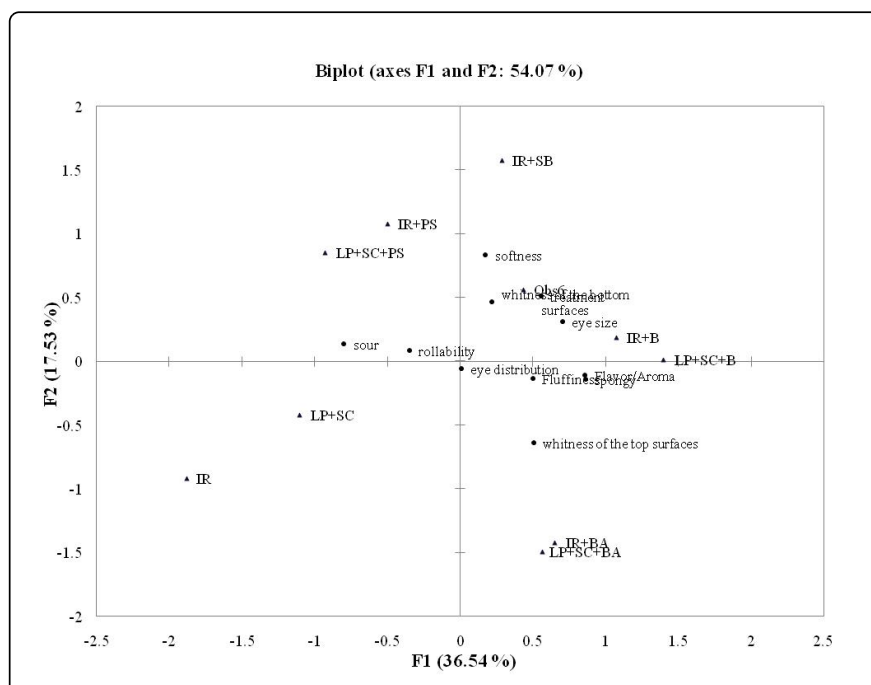

Figure 1: PCA biplot ('sensory map') showing the sensory attributes of rice injera fermented with $L$. plantarum $+S$. cerevisiae starter culture and irsho with and without chemical preservatives added. Key: $\mathrm{IR}=$ rice injera from traditional starter $(\mathrm{Irsho}), \mathrm{LP}+\mathrm{SC}=$ rice injera with $L$. plantarum and $S$. cerevisiae starter culture, IR $+\mathrm{BA}=$ rice injera from traditional starter (Irsho) with $0.1 \%$ benzoic acid, $\mathrm{LP}+\mathrm{SC}+\mathrm{BA}=$ rice injera with $L$. plantarum and $S$. cerevisiae starter culture with $0.1 \%$ benzoic acid, $\mathrm{SB}=$ rice injera from traditional starter (Irsho) with $0.1 \%$ sodium benzoate, LP+SC $+\mathrm{SB}=$ rice injera with $L$. plantarum and $S$. cerevisiae starter culture with $0.1 \%$ sodium benzoate, $\mathrm{IR}+\mathrm{PS}=$ rice injera from traditional starter (Irsho) with $0.2 \%$ potassium sorbate, $\mathrm{LP}+\mathrm{SC}+\mathrm{PS}=$ rice injera with $L$. plantarum and $S$. cerevisiae starter culture with $0.2 \%$ potassium sorbate, $\mathrm{B}=$ rice injera from traditional starter (Irsho) with $0.2 \%$ the blend of the three preservatives, $\mathrm{LP}+\mathrm{SC}+\mathrm{B}=$ rice injera with $L$. plantarum and $S$. cerevisiae starter culture with $0.2 \%$ the blend of the three preservatives. Factors affecting the generation of electricity. 
Citation: Hassen Y, Mukisa IM, Kurabachew H (2017) The Effect of Selected Chemical Preservatives and Starter Cultures on the Sensory Characteristics and Shelf Life of Rice Injera. J Bioprocess Biotech 8: 313. doi:10.4172/2155-9821.1000313

Page 4 of 7

\section{Consumer acceptability}

The effect of chemical preservatives on the sensory acceptability of rice injera is presented in Table 2. All the samples of rice injera scored from 7.82 to 8.79 (like very much). The preservatives had a significant effect $(\mathrm{p}<0.05)$ on the scores of overall acceptability. The rice injera made from the $0.2 \%$ blend of the three preservatives $(0.1 \%$ benzoic acid, $0.1 \%$ sodium benzoate and $0.2 \%$ potassium sorbate) and $0.2 \%$ potassium sorbate had the highest overall acceptability scores: $8.79 \pm$ 0.91 (like extremely) and $8.47 \pm 0.72$ (like very much). Control rice injera (fermented with irsho and with no preservatives and that fermented with the $L$. plantarum and $S$. cerevisiae (without preservatives) scored the least in overall acceptability: $7.84 \pm 0.85$ and $7.82 \pm 1.21$ respectively. Rice injera made with $L$. plantarum $+S$. cerevisiae and preserved with $0.2 \%$ of the blend of the three $(0.1 \%$ benzoic acid, $0.1 \%$ sodium benzoate and $0.2 \%$ potassium sorbate) and rice injera fermented with $L$. plantarum $+S$. cerevisiae and with $0.1 \%$ benzoic acid.

Do not affect the over acceptability of the injera or it has no negative impact the sensory attributes.

\begin{tabular}{|l|l|l|l|l|l|}
\hline Treatment & Appearance & Taste & Aroma & Mouth feel & Overall acceptance \\
\hline Irsho & $7.43 \pm 0.87 \mathrm{~g}$ & $7.06 \pm 1.45 \mathrm{~h}$ & $7.27 \pm 1.26 \mathrm{f}$ & $7.24 \pm 1.13 \mathrm{~h}$ & $7.84 \pm 0.85 \mathrm{fg}$ \\
\hline LP+SC & $7.52 \pm 1.24 \mathrm{f}$ & $7.62 \pm 1.22 \mathrm{~d}$ & $7.67 \pm 0.97 \mathrm{dc}$ & $7.34 \pm 1.47 \mathrm{~g}$ & $7.82 \pm 1.21 \mathrm{~g}$ \\
\hline Irsho+0.1\% BA & $7.73 \pm 0.98 \mathrm{e}$ & $7.59 \pm 1.24 \mathrm{~d}$ & $7.48 \pm 1.28 \mathrm{e}$ & $7.67 \pm 1.90 \mathrm{~d}$ & $8.04 \pm 0.88 \mathrm{e}$ \\
\hline LP+SC+0.1\% BA & $8.12 \pm 1.06 \mathrm{~d}$ & $7.92 \pm 1.11 \mathrm{c}$ & $7.61 \pm 1.44 \mathrm{~d}$ & $7.99 \pm 1.55 \mathrm{c}$ & $8.23 \pm 0.90 \mathrm{~d}$ \\
\hline Irsho+0.1\% SB & $7.46 \pm 1.17 \mathrm{fg}$ & $7.38 \pm 1.36 \mathrm{f}$ & $7.13 \pm 1.47 \mathrm{~g}$ & $7.43 \pm 1.20 \mathrm{f}$ & $7.89 \pm 0.94 \mathrm{f}$ \\
\hline LP+SC0.1\% SB & $8.27 \pm 0.82 \mathrm{bc}$ & $8.03 \pm 0.95 \mathrm{~b}$ & $7.77 \pm 1.39 \mathrm{c}$ & $8.10 \pm 1.05 \mathrm{~b}$ & $8.33 \pm 0.79 \mathrm{c}$ \\
\hline Irsho+0.2\% PS & $7.66 \pm 1.15 \mathrm{e}$ & $7.48 \pm 1.35 \mathrm{e}$ & $7.22 \pm 1.51 \mathrm{fg}$ & $7.56 \pm 1.21 \mathrm{e}$ & $7.99 \pm 0.95 \mathrm{e}$ \\
\hline LP+SC+0.2\% PS & $8.30 \pm 0.90 \mathrm{ab}$ & $8.27 \pm 0.89 \mathrm{a}$ & $8.00 \pm 1.27 \mathrm{~b}$ & $8.30 \pm 0.94 \mathrm{a}$ & $8.47 \pm 0.72 \mathrm{~b}$ \\
\hline Irsho+0.2\% (BA+SB+PS) & $7.41 \pm 0.96 \mathrm{~g}$ & $7.26 \pm 1.45 \mathrm{~g}$ & $7.23 \pm 1.32 \mathrm{c}$ & $7.29 \pm 1.19 \mathrm{gh}$ & $8.79 \pm 0.91 \mathrm{~g}$ \\
\hline LP+SC+0.2(BA+SB+PS) & $8.27 \pm 0.89 \mathrm{bc}$ & $8.03 \pm 0.95 \mathrm{~b}$ & $7.73 \pm 1.37 \mathrm{c}$ & $8.10 \pm 1.05 \mathrm{~b}$ & $8.37 \pm 0.75 \mathrm{abc}$ \\
\hline
\end{tabular}

Table 2: Effect of different preservatives on the sensory acceptability of rice injera. Values are mean standard deviation ( $\mathrm{n}=30)$. Values in the same column with different superscripts $(\mathrm{a}-\mathrm{h})$ are significantly different $(\mathrm{p}<0.05)$. Where, $\mathrm{LP}=$ Lactobacillus plantarum, $\mathrm{SC}=$ Saccharomyces cerevisiae, (9="like extremely"; 8 ="like very much"; 7="like moderately"; 6="like slightly"; 5="neither like nor dislike"; 4="dislike slightly"; 3="dislike moderately"; 2 ="dislike very much"; 1="dislike extremely").

\section{The effect of preservatives on the shelf life of injera}

The effect of chemical preservatives on the shelf life of injera is shown in Table 3. The use of preservatives led to an increase in shelf life from 2-3 days to 6-8 days for samples fermented for $24 \mathrm{~h}$ and from 3-4 to 7-9 for samples fermented for 48 hours. Increasing fermentation time from 24 to $48 \mathrm{~h}$ resulted in a 1-2 day increase in shelf life. The lowest shelf life (2-3 days was observed in the rice injera prepared as a control without addition of preservatives. The longest shelf life (9-10 days) was observed for injera prepared from $0.2 \%$ of the blend of the three preservatives $(0.1 \%$ benzoic acid, $0.1 \%$ sodium benzoate and $0.2 \%$ potassium sorbate) in $1: 1: 1$ which is $9-10$ days.

\begin{tabular}{|l|l|l|}
\hline Treatment & Observed shelf life (days) & $\mathbf{4 8} \mathbf{h}$ fermented samples \\
\hline Irsho & $\mathbf{2 4} \mathbf{h}$ fermented samples & 43193 \\
\hline LP+SC & 43161 & 43193 \\
\hline Irsho+0.1BA & 43161 & 43319 \\
\hline LP+SC+0.1BA & 43287 & 43319 \\
\hline Irsho $+0.1 \%$ SB & 43287 & 43351 \\
\hline LP+SC $+0.1 \%$ SB & 43319 & 43351 \\
\hline Irsho+0.2\% PS & 43319 & 43351 \\
\hline LP+SC+0.2\% PS & 43319 & 43351 \\
\hline
\end{tabular}


Page 5 of 7

\begin{tabular}{|l|l|l|}
\hline Irsho+0.2\% (BA+SB+PS) & 43319 & 43382 \\
\hline LP+SC+0.2\% (BA+SB+PS) & 43319 & 43382 \\
\hline
\end{tabular}

Table 3: Effect of preservatives on the shelf life of rice injera baked after 24 and $48 \mathrm{~h}$ of fermentation; where, LP=Lactobacillus plantarum, $\mathrm{SC}=$ Saccharomyces cerevisiae, Irsho (traditional starter back slopping), BA=Benzoic acid, SB=sodium benzoate, PS=potassium sorbate." Shelf life was defined as the time taken before observing mould growth on the surface of the product.

\section{Discussion}

\section{Descriptive sensory characteristic of rice injera}

The characteristics include whiteness of the top and bottom surface, eye size, eye distribution, softness, fluffiness, roll ability, aroma and Sourness are the major descriptors of rice injera. This description is similar to that of teff injera which is characterized by porosity and having numerous eyes. The description is also similar with that of sorghum injera. A higher number of medium eyes are a very desirable attribute of an attractive injera. Injera is characterized by having "eyes" (honeycomblike holes) in its top surface [13]. These are produced due to the production and escape of carbon dioxide during fennentation and baking, respectively.

From the results (Figure 1), attributes found close together on the biplot are positively correlated across the products and attributes found at opposite ends of the biplot are negatively correlated across the products. Attributes found in the same location as a product tend to be characteristic for that product. This observation has been reported in the use of principal component analysis (PCA) to characterize beef. In this study Eye size and flavor/aroma were highly correlated in 24 hours fermented batter of rice injera. Fluffiness and spongy characteristics are also highly correlated in 24 hours fermented batter rice injera. Softness was very closely associated with roll ability which observation agrees with the tendency of soft injera to roll easily. This in line with Yetneberk [14]. Who reported that softness was very closely associated with roll ability. This agrees with the observed tendency of soft injera to roll easily. Both characteristics (fluffiness and spongy are considered important eating qualities of injera. Good injera is soft and roll able to wrap and hold the sauce (wot) during consumption [15]. A good injera is also spongy and can be folded without cracking. The color of the rice injera in the present study was white in both top and bottom surfaces which is desirable by most of the consumers. This is in agreement with ICRISAT (1982) which stated that the most consumers preferred injera which is whitish or cream in color. The rice injera made from $24 \mathrm{~h}$ fermented batter in the present study was slightly sour which the characteristic taste is of injure. Good injera must be slightly sour.

The color of the rice injera in the present study was white in both top and bottom surfaces which is desirable by most if the consumers. This is in agreement with ICRISAT which stated that the most consumers preferred injera which is whitish or cream in color. The rice injera made from $24 \mathrm{~h}$ fermented batter in the present study was slightly sour which the characteristic taste of injera is. Good injera must be slightly sour (ICRISAT).

The treatments on the right side of the coordinate (irsho+benzoic acid) and ( $L$. plantarum $+S$. cerevisiae+Benzoic acid, Irsho+benzoic acid) and (L. plantarum $+S$. cerevisiae+Benzoic acid) in $24 \mathrm{~h}$ fermented rice batter injera which is rice injera baked from a mixed culture of $L$. plantarum, and $S$. cereviseae and mixed preservatives has good sensory attributes.
The position of the products and of the variables in this twodimensional biplot shows which attributes are associated with which products, and essentially is a 'sensory map' of the products.

Controlled fermentation by $L$. plantarum, and $S$. cerevisiae produced injera with the desirable sensory quality. The finding of this study in agreement with Mogessie who stated that the proliferation of lactic acid bacteria during fermentation of teff for injera making produces the necessary metabolites for flavor and taste [15]. Glover et al. also reported that starter culture combinations of LAB and yeast produce better quality products with desirable aroma and flavor compounds. The yeast starter is important for the production of carbon dioxide which contributes to creation of eyes in injera. Although yeasts have the primary leavening role, lactic acid bacteria with trophic and non-trophic relationships; produce important flavor components. In a previous study, Berhanu Abegaz Gashe et al. stated that initial fermentation activity was carried out by a group of Gramnegative aerogenic groups. The activity of these groups resulted in excessive evolution of gas and 'dough rises'.

\section{Consumer acceptability}

The findings of this study showed that the rice injera prepared with chemical preservatives were not affecting the overall acceptability. Concentration and synergistic addition of $0.2 \%$ of the blend of the three preservatives $(0.1 \%$ benzoic acid, $0.1 \%$ sodium benzoate and $0.2 \%$ potassium sorbate) in 1:1:1 and sodium benzoate seem to have no effect on their ability to act differently for the change on overall sensory acceptability of the rice injera. Ogiehor and Ikenebomeh stated that gari (fermented product from cassava) treated with sodium benzoate were highly acceptable. In the current study the combination of $L$. plantarum and Saccharomyces cerevisae extended the shelf life of bread up to 10 days, which may be due to the production of lactic acid by these organisms from carbohydrate and the resultant decrease in $\mathrm{pH}$ [16]. Apart from lactic acid produced by these organisms, other metabolites such as diacety and, hydrogen peroxide were also produced as reported by Ogunbanwo et al., which could act in synergy to prolong the shelf life of the injera.

In the study on Agbarati (an indigenous meat analogue produced mainly in the rural areas of South Eastern Nigeria) the use of chemical preservative in controlled concentrations was considered adequate to prolong shelf life of the Agbarati sampler relative to the unprotected or untreated control samples [16]. This is so since it has no adverse effect on the quality and sensory attributes of the stored Agbarati samples [17] also observed that the action of potassium sorbate, potassium metabisulphite and sodium benzoate, retains overall acceptability, nutrients stability and reduces microbial load of mango. The findings of this study showed that the product preserved with chemical preservatives $(0.1 \%$ benzoic acid, $0.1 \%$ sodium benzoate and $0.2 \%$ potassium sorbate) has no effect on sensory attributes of rice injera. Hashmi et al. observed that potassium sorbate, potassium metabisulphite, sodium benzoate separately or in combination with 
other chemical preservatives used for the improvement of sensory characteristics, control microbes and retain overall acceptability.

Numerous studies have confirmed the effect of adding chemical preservatives and subsequent storage at ambient temperature of the samples on the organoleptic characteristics. Generally, from the findings recorded in the results as discussed above, the chemical preservatives and their concentrations used in the current study also do not affect the taste of the rice injera. Sodium benzoate and benzoic acid are used at low level to avoid off-flavor. Benzoic acid and its sodium salt along with the esters of p-hydroxybenzoic acid (parabens) are permissible in foods at up to $0.1 \%$ level [18]. Maximum levels recommended by the Food and Drug Administration of USA for potassium sorbate is $0.2 \%$ in food. The use of these preservatives should not exceed the maximum level allowed by law. Benzoic acid has been found to cause no deleterious effect when used in small amounts. It is also readily eliminated from the body after conjugation with glycine to form hippuric acid.

\section{Effect of preservatives and starter culture on the shelf life of rice injera}

Results from this study indicated that addition of preservatives $(0.2 \%$ of the blend of the three preservatives $(0.1 \%$ benzoic acid, $0.1 \%$ sodium benzoate and $0.2 \%$ potassium sorbate) in 1:1:1 increased the shelf life of rice injera from 2-3 to 9-10 days. Results from this study are in agreement with that of Ashagre. Ashagre in his study on improvement of teff injera shelf life through the use of chemical preservatives indicated that the use of antifungals activities of the preservatives $0.1 \%$ benzoic acid, $0.1 \%$ sodium benzoate and $0.2 \%$ potassium sorbate prolonged the shelf life of injera for up to 12 days. Preservatives are added to prevent the growth of undesirable microorganisms, food spoilers and foodborne pathogens. The selection and combination of the right preservative is essential to obtain the best results in shelf life.

The combined effect of the preservatives $(0.1 \%$ benzoic acid, $0.1 \%$ sodium benzoate and $0.2 \%$ potassium sorbate) in this study is agreement with Martinez et al. which indicated the combined action of two or more preservatives is more effective in inhibiting the growth of microorganisms, which could be due to a synergistic effect among the preservatives. This is also in line with Ashagre findings in which found out the blend of the preservatives $(0.1 \%$ benzoic acid, $0.1 \%$ sodium benzoate, $0.2 \%$ potassiumand $0.3 \%$ calcium propionate) is effective in prolonging the shelf life of injera up to 8 days. In case of the $24 \mathrm{~h}$ fermented rice injera the combinations of the three preservatives showed better shelf life than the other treatments. Mihyar et al. reported that potassium sorbate and sodium benzoate at levels of 0.025 and $>0.04 \%$ extended the shelf life of labaneh (concentrated yoghurt) from 14 days to 21 days, respectively. In case of freshly made injera, it is free of fungi but the large surface area of the flat bread provides high probability of fungal contamination from the air. Mohammed et al. also indicated addition of $0.05 \%$ and $0.1 \%$ potassium sorbate and $0.1 \%$ and $0.15 \%$ of sodium benzoate are the most effective preservative increased the shelf life of hummus to 35 and 49 days, respectively, one of the most popular traditional foods in Middle East countries such as Jordan, Syria and Lebanon which is prepared from dried chickpeas (Cicer arietinum L.) and tahina (an oily viscous fluid obtained by milling dehulled roasted white sesame seeds). Results of the current study on rice injera agree with these findings in respect to effective inhibition of potassium sorbate and sodium benzoate of yeasts and molds. Both of potassium sorbate and sodium benzoate are well- known for their higher effectiveness against yeasts and molds than against bacteria, and the preservative potassium sorbate has a more inhibitory effect than sodium benzoate.

The effectiveness of the organic acids used in this study against mould growth generally increased in the order of benzoic acid<sodium benzoate which is in agreement with the results of Gould s who indicated that the order reflects their increasing lipophilicity. Benzoic acid and its salt (sodium benzoate) were the most effective preservatives in delaying the spoilage of injera as compared to the other preservatives tested ( 0.1 benzoic caid and potassium sorbate). This could be due to the fact that antimicrobial activity of benzoic acid is essentially in the undissociated form $(\mathrm{pKa}=4.2)$ which has the ability to interface with membrane energetic. Moreover, benzoic acid is relatively acidic, which supports the inhibition of the growth of spoilage moulds. Further, it can be speculated that the higher effectiveness of sodium benzoate as compared to benzoic acid could be due to the fact that the salt is 200 times more soluble than the acid.

\section{Conclusion}

Nine descriptors/attributes describing appearance, aroma/odor, taste, and texture were generatedto characterizing the sensory properties of rice injera. Softness, reliability, fluffiness, eye size, whiteness of top and bottom surfaces and slight sour taste are main characterstics descriptors of rice injera. Softness, fluffiness, sponginess, slightly sourness and eye size are attributes associated with quality of rice injera. The findings of this study showed that the rice injera prepared with food preservatives $(0.1$ benzoic acid, $0.2 \%$ potassium sorbate, $0.1 \%$ sodium benzoate and $0.2 \%$ of the three preservatives retain maximum overall acceptability. Rice injera produced under controlled fermentation $L$. plantarum, and $S$. cereviseae produce injera with better sensory quality. The blend preservatives of $0.2 \%$ potassium sorbate, $0.1 \%$ sodium benzoate and $0.1 \%$ benzoic acid was the most effective of mono preservative followed by $0.1 \%$ sodium benzoate and $0.2 \%$ potassium sorbate. The preservatives used in this study prolonged the shelf life of rice injera for up to 10 days. Therefore, a starter combination of $L$. plantarum $+S$. cerevisiae together with $0.2 \%$ of the blend of the preservatives $(0.1 \%$ benzoic acid $+0.1 \%$ sodium benzoate $+0.2 \%$ potassium sorbate) in 1:1:1 ratio preservatives can be used to produce acceptable injera with a shelf life of 10 days at room temperature. More detailed work on the physicochemical properties of the rice, maize and wheat flours should be conducted and correlated with injera quality.

\section{References}

1. Akpan UG, Kovo AS (2005) Preservation of passion fruit juice. Leonardo J Sci 7: 17-22.

2. Ashagrie Z, Abate (2012) Improvement of injera shelf life through the use of chemical preservatives. African J Food Agri, Nutri Develop 12: 42-84.

3. Askal D, Kebede A (2013) Isolation characterization and identification of lactic acid bacteria and yeast involved in fermentation of Teff (EragrostisTef) Batter. Int J Advan Res Bio Sci 1: 36-44.

4. Carpenter RP, Lyon HD, Hasdell AT (2000) Guidelines for Sensory Analysis in Food Product Development and Quality Control. Aspen Publishers, USA.

5. Cross HR, Moen R, Stanfield MS (1978) Training and testing of judges for sensory analysis of meat quality. J Food Tech 32: 48-54.

6. Destefanis G, Barge MT, Brugiapaglia A, Tassone S (2000) The use of principal component analysis (PCA) to characterize beef. Meat Science 56: $255-259$ 
Citation: Hassen Y, Mukisa IM, Kurabachew H (2017) The Effect of Selected Chemical Preservatives and Starter Cultures on the Sensory Characteristics and Shelf Life of Rice Injera. J Bioprocess Biotech 8: 313. doi:10.4172/2155-9821.1000313

Page 7 of 7

7. Doughari JH, Alabi G, Elmahmood AM (2007) Effect of some chemical preservatives on the shelf life of sobo drink. Afric J Micro 2: 37-41.

8. Gould GW (1999) Methods of preservation and extension of shelf life. Inter J Food Micro 33: 51-64.

9. International Commission on Microbiological Specifications for Foods (2005) Microorganisms in Foods-6. Microbial Ecology of Food Commodities. 2nd edn, KlumerAcademic Publisher, New York.

10. Jay JM (2000) Modern Food Microbiology. Aspen Publishers, Maryland.

11. Meilgaard M, Ciille GV, Carr BT (1999) Sensory Evaluation Techniques. 3rd edn, CRC Press Inc, Boca Raton, Florida, US.

12. Mogessie A (2006) A Review on the microbiology of indigenous fermented foods and beverages of Ethiopia. Ethiop J Biol Sci 5: 189-245.

13. Mihyar GF, Yamani MI, Al-Sa'ed AK (1997) Resistance of yeast flora of labaneh to PS and SB. J Dairy Sci 80: 2304-2309.
14. Mohammed IY, Ghadeer FM (2011) Effect of Chemical Preservatives on the Shelf Life of Hummus during Different Storage Temperatures. Jordan J Agri Sci 7: 123-132.

15. Mpountoukas P, Vantarakis A, Sivridis E, Lialiaris T (2008) Cytogenetic study in cultured human lymphocytes treated with three commonly used preservatives. Food Chem Toxic 6: 2390-2393.

16. Santini AO, Pezza HR, Filho OC, Sequinel R, Pezza L (2009) Potentiometric sensor for sorbic acid determination in food products. Food 14: 45-54.

17. Senayit Y, de Kock H, Lloyd W, Rooney R (2004) Effects of Sorghum Cultivar on Injera Quality. Cereal Chem 81:314-321.

18. Tfouni SAV, Toledo MCF (2002) Determination of benzoic and sorbic acids in Brazilian food. Food Control 13: 117-123. 